\title{
Bilateral Hypoglossal Nerve Palsy in a Patient with Breast Cancer
}

Gina Hyun*, Louis K Chen and Blair Glasgo Walter Reed National Military Medical Center, Bethesda

*Corresponding author: Gina Hyun, Walter Reed National Military Medical Center, Bethesda

Received: October 15, 2018; Accepted: October 26, 2018; Published: November 02, 2018

\begin{abstract}
We describe a case of a 64-year-old woman who presented with isolated bilateral hypoglossal nerve palsy. After an extensive workup including an unremarkable Computed Tomography (CT) scan, she was found to have skull base metastasis from breast cancer on thin-slice Magnetic Resonance Imaging (MRI). This is the first reported case of isolated bilateral hypoglossal nerve palsy and demonstrates that thin-slice MRI provides.
\end{abstract}

Keywords: Hypoglossal nerve palsy; Cranial nerve palsy; Brain metastasis; Breast cancer

\section{Introduction}

Isolated hypoglossal nerve palsies are rare and may portend an underlying malignancy. Typically, they present with unilateral tongue deviation as the metastatic focus localizes to one hypoglossal canal.

Here, we report a 64-year-old woman who presented with poor oral intake and was subsequently found to have bilateral hypoglossal nerve palsy shortly after being diagnosed with metastatic breast cancer.

\section{Case Presentation}

A 64-year-old woman was diagnosed with stage IIIA (T3N1M0) left breast Invasive Ductal Carcinoma (IDC) in 2014. Following neoadjuvant chemotherapy with docetaxel, carboplatin, trastuzumab, and pertuzumab, she underwent lumpectomy and local radiation therapy. Three years later, she was diagnosed with recurrent stage IV breast cancer. Shortly after diagnosis she was admitted with poor oral intake, which she attributed to new onset "tongue swelling" after a contrasted imaging study, as she had experienced a similar sensation in her tongue after prior contrasted imaging studies.

Physical examination showed an immobile tongue without lateral deviation or obvious swelling. The remainder of her neurologic exam was nonfocal. While an initial non-contrast head CT scan was unremarkable, an MRI brain revealed a $0.6 \mathrm{~cm}$ ring-enhancing lesion in the pons with surrounding edema (Figure 1). The clinical significance of this lesion was suspect given its location and the bilateral nature of her palsy. After empiric therapy for oral thrush, an unrevealing allergy evaluation, and a speech pathology evaluation, further imaging was deemed necessary to search for a lesion causing her isolated bilateral tongue palsy. A repeat MRI brain with thinner slices through the base of the skull revealed osseous tumor burden at the level of the occipital and sphenoid skull base specifically involving the bilateral hypoglossal canals (Figure 2).

Further along in the work up, the patient developed mild left facial weakness with vocal hoarseness and was found to have oropharyngeal dysphagia on a modified barium swallow study.

The patient was treated with palliative whole-brain radiation therapy and dexamethasone with improvement in her tongue mobility, but she unfortunately expired from complications of her disease process one month later.

\section{Discussion}

Breast cancer is a leading cause of CNS metastasis, which can be further categorized into three patterns: leptomeningeal, intracranial and spinal cord. Cranial nerve palsies, a clinical sign of leptomeningeal metastasis, often carries a poor prognosis with mean survival rate of 20 months [2]. In cases of CNS metastasis secondary to breast cancer, one study found that cranial nerve palsies occurred approximate 30 percent of the time with nerves $\mathrm{V}$ and VII being the most commonly affected [3].

Hypoglossal nerve palsy, especially bilateral, is a rare phenomenon that warrants careful evaluation. The most common etiologies of isolated hypoglossal nerve palsy include recent surgery, radiation, and trauma. While half of all other cases are idiopathic, approximately 20 percent of cases are secondary to malignancy [4]. Bilateral nerve palsy, as in this patient's case, warrants a careful search for other neurologic

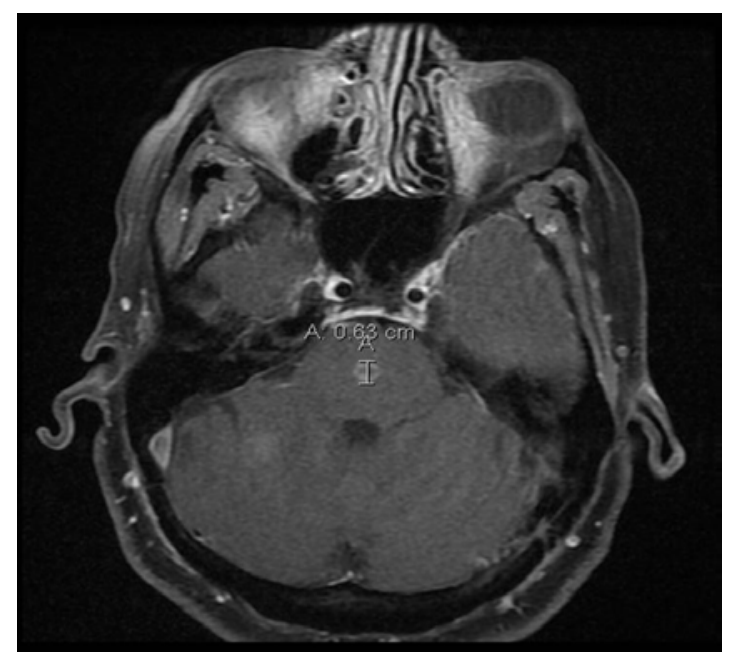

Figure 1: This figure shows an MRI brain revealed a $0.6 \mathrm{~cm}$ ring-enhancing lesion in the pons with surrounding edema. 


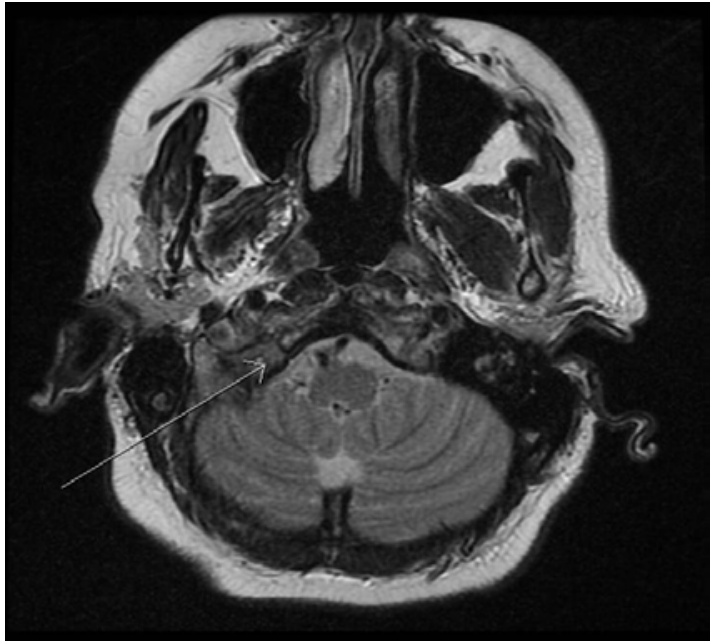

Figure 2: The shows the occipital and sphenoid skull base specifically involving the bilateral hypoglossal canals.

deficits and directed imaging looking for metastatic disease to the brain and calvarium given the nerve's location in the hypoglossal canal. As suggested by other studies $[1,5,6]$, an MRI scan with thin slices provides the most diagnostic information.

We believe that our patient's hypoglossal nerve palsy was directly caused by metastases to the skull base, but the corticobulbar tract that decussates in the pons may also have been culpable given her subsequent bulbar weakness and lesion in the pons.

\section{Conclusion}

Metastatic breast cancer may present with isolated hypoglossal or other cranial nerve palsies and a thorough neurologic exam with thin-slice MRI scan is warranted in the workup.

\section{References}

1. Khoo SG, Ullah I, Wallis F, Fenton JE. Isolated hypoglossal nerve palsy: A harbinger of malignancy. J Laryngol Otol. 2007; 121: 803-805.

2. Hall SM, Buzdar AU, Blumenschein GR. Cranial nerve palsies in metastatic breast cancer due to osseous metastasis without intracranial involvement. Cancer. 1983; 52: 180-184.

3. Carty NJ, Foggitt A, Hamilton CR, Royle GT, Taylor I. Patterns of clinical metastasis in breast cancer: An analysis of 100 patients. Eur J Surg Oncol. 1995; 21: 607-608.

4. Combarros $\mathrm{O}$, Alvarez de arcaya A, Berciano J. Isolated unilateral hypoglossa nerve palsy: nine cases. J Neurol. 1998; 245: 98-100.

5. Fujii $\mathrm{H}$, Ohtsuki T, Takeda I, Hosomi N, Matsumoto M. Isolated unilatera hypoglossal nerve paralysis caused by internal carotid artery dissection. J Stroke Cerebrovasc Dis. 2014; 23: e405-e406.

6. Hashimoto M, Ohtsuka K. Compressive lesions of the abducens nerve in the subarachnoid space disclosed by thin-slice magnetic resonance imaging. Ophthalmologica. 1998; 212: 188-189.
Ann Hematol Oncol - Volume 5 Issue 7 - 2018

ISSN : 2375-7965 | www.austinpublishing group.com Hyun et al. ( ) All rights are reserved
Citation: Hyun G, Chen LK and Glasgo B. Bilateral Hypoglossal Nerve Palsy in a Patient with Breast Cancer. Ann Hematol Oncol. 2018; 5(7): 1219. 